Received: 2 June 2017

Accepted: 8 January 2018

Published online: 22 January 2018

\section{Homeolog expression analysis in an allotriploid non-model crop via integration of transcriptomics and proteomics}

\author{
Jelle van Wesemael ${ }^{1}$, Yann Hueber ${ }^{2}$, Ewaut Kissel ${ }^{1}{ }^{1}$, Nádia Campos ${ }^{1}$, Rony Swennen ${ }^{1,3,4} \&$ \\ Sebastien Carpentier ${ }^{1,3,5}$
}

The fate of doubled genes, from allopolyploid or autopolyploid origin, is controlled at multiple levels, resulting in the modern day cultivars. We studied the root growth of 3 different triploid banana cultivars under control and osmotic stress conditions. The root growth of the allopolyploid ABB cultivar was $42 \%$ higher under control and $61 \%$ higher under osmotic stress. By integrating transcriptomics and proteomics, we studied the gene expression of all 3 cultivars, resulting in 2,749 identified root proteins. 383 gene loci displayed genotype specific differential expression whereof 252 showed at least one Single Amino Acid Polymorphism (SAAP). In the ABB cultivar, allele expressions supposedly follow a $1 / 3$ and $2 / 3$ pattern for respectively the $A$ and the $B$ allele. Using transcriptome read alignment to assess the homeoallelic contribution we found that $63 \%$ of the allele specific genes deviated from this expectation. 32 gene loci even did not express the $A$ allele. The identified ABB allele- specific proteins correlate well with the observed growth phenotype as they are enriched in energy related functions such as ATP metabolic processes, nicotinamide nucleotide metabolic processes, and glycolysis.

Bananas and plantains (Musa spp.) are a major allopolyploid crop with a yearly production of \pm 145 million tonnes (2014, FAOstat), spread over the (sub-)humid tropics, an estimated $85 \%$ of which coming from smallholder plots in the developing world ${ }^{1}$. Banana demands a lot of water and drought stress is the major limiting abiotic stress factor ${ }^{2}$. For example in the East-African highlands, where the crop evapotranspiration is between 1200 and $1300 \mathrm{~mm}$ per year, there is an $8-10 \%$ yield decline per $100 \mathrm{~mm}$ water that could not be transpired ${ }^{2}$. In the $21^{\text {st }}$ century, agriculture is challenged to feed an increasing population while minimizing unsustainable usage of (natural) resources. This means that crops (including banana as a top ten staple food crop) need to be better adapted to a changing and increasingly variable environment ${ }^{3-6}$. Fitness, survival, and agricultural productivity result from the plant genotype, environment interaction and Management $(\mathrm{G} \times \mathrm{E} \times \mathrm{M})$. Flexibility of plants towards the environment is naturally determined by genetic diversity $(\mathrm{G})$, and a deeper understanding thereof towards the phenotype is a priority ${ }^{7,8}$. It is a major objective for crop scientists to identify sources of natural variation with potential to rise the tolerance towards unfavourable (a)biotic constraints while minimizing the yield penalty ${ }^{8}$. The majority of plant research has focused on model species (Arabidopsis thaliana) and major, sequenced crops (e.g. Oryza sativa, Zea mays, Glycine max). However, many other important food, feed, or energy crops and their wild relatives are highly relevant in the quest for sustainable cultivars. Among these are numerous polyploid crops with complex heterologous genomes.

Modern Musa cultivars are triploid, hybrid crosses characterized by high levels of gene flow between members of the Musaceae family, mainly: Musa acuminata (A genome) and Musa balbisiana (B genome) ${ }^{9,10}$. The modern, seedless, vegetative clones originate from initial sexual reproductions between these seedbearing progenitor species ${ }^{11,12}$. Different levels of ancestor (A or B) genome contribution are noted. After the polyploidization,

${ }^{1}$ Laboratory of Tropical Crop Improvement, KU Leuven, Willem Decroylaan 42, Leuven, Belgium. ${ }^{2}$ Bioversity International, Parc Scientifique Argropolis II, Montpellier, France. ${ }^{3}$ Bioversity International, Willem Decroylaan 42, Leuven, Belgium. ${ }^{4}$ International Institute for Tropical Agriculture, C/O Nelson Mandela Institute of Science and technology, P.O. Box 44, Arusha, Tanzania. ${ }^{5}$ Facility for SYstems BIOlogy based MAss spectrometry, Herestraat 49, Leuven, Belgium. Correspondence and requests for materials should be addressed to S.C. (email: sebastien. carpentier@kuleuven.be) 
and consequent sterilization, genetic diversity was only enhanced by mutation ${ }^{9}$. Reference sequences have been published for the Musa acuminata (A) genome, and a draft reference sequence for the Musa balbisiana (B) genome $^{13-15}$. Efforts have also been made to sequence crop wild relative species of banana (Musa itinerans) since these may be informative for modern banana cultivars ${ }^{16}$. The dissimilarity between the Musa acuminata and the draft Musa balbisiana reference genomes is 1 SNP per $39 \mathrm{bp}^{13}$.

Polyploid crops such as banana are characterized by a broad genetic diversity, acquired by genome merging and doubling ${ }^{17}$. Autopolyploids are constituted by chromosome sets of closely related populations, while allopolyploids result from interspecific hybridization ${ }^{18,19}$. The genome redundancy provides means for genetic novelty and adaptability towards environmental cues ${ }^{20}$. Gene loss or silencing, neo- and/or sub-functionalization, intergenomic transfer, allele dominance/co-dominance, differences in transcription/translation efficiency and post translational modifications, exemplify how the genome, transcriptome, and proteome are regulated following polyploidization events ${ }^{18-20}$. The allo-/and autopolyploid genome is thus a patchwork of gene variants enabling many genotype $\times$ environment interactions. Features unique for a preferential phenotype at the gene variant level are most probable candidate markers controlling the observed phenotype.

Advances in next- generation sequencing techniques enable high- resolution linkage studies between gene variants and traits of interest (Genome- Wide Association Studies, GWAS). However, the high degree of heterozygosity and the amount of replicated sequence reads in polyploid crops is a great challenge for effective single nucleotide polymorphism (SNP) calling ${ }^{8,21}$. In banana GWAS has been successfully applied in diploids for the seedless phenotype, a trait underpinned by a limited number of genes ${ }^{12}$. But as stated, this approach on the allopolyploid triploids for a complex multi gene trait is cumbersome. The link between gene or protein abundance and SNP or single amino acid polymorphisms (SAAP) and a preferential phenotype is more stringent with knowledge of the tissue, time point and environmental condition of the gene/protein activity, i.e. using functional genomics (transcriptomics and/or proteomics) ${ }^{22}$. RNA sequencing effectively combines gene expression quantification with gene sequencing and allows SNP calling. Yet, most current day read mapping software is written for diploid species and have difficulties to process complex (polyploid) genomes. The read mapping efficiency to the reference genome might be biased, and the degree of heterozygosity greatly increases computational effort, hampering quantitative results per genome ${ }^{23}$. Consequently the RNA reads are not separated and traced back to their (sub-) genome. Algorithms like PolyCat and HANDS2 process reads based on classification towards their genome of origin, but heavily depend on the presence and the quality of reference genomes ${ }^{23,24}$. Since not all cultivars of interest carry the reference sequence, mapping efficiency biases can still occur when one reference genome is more closely linked to a constituting genome than the other ${ }^{23}$.

Proteomics is a bottom-up, specific technique allowing to pick up and quantify the actual differential product as it is controlling the phenotype. Gel- based proteomics is very useful to identify allele- specific protein isoforms in a non-model crop ${ }^{25}$. This is exemplified in the HSP70 protein family in banana ${ }^{26}$. But, for multigenic traits, like tolerance to abiotic stress, it is important to get a broader picture. Via LC-MSMS multiple allele- specific products (tryptic specific peptides) are quantified without required prior knowledge ${ }^{27}$. Peptide identification is greatly enhanced with the availability of a reference genome. In reality, in non-model crops the database of the nearest related taxon can also be used. In essence LC-MSMS is suitable for the study of specific allelic contributions towards a preferential phenotype in polyploid non-model crops, eg. Hu et al ${ }^{28}$ discovered 558 allele- specific protein isoforms in Gossypium using iTRAQ.

This study aims to select alleles that are correlated to drought stress in banana (Musa spp.), an allopolyploid, non-model crop. Therefore we rely on a lab model mimicking drought stress using an osmotic stress agent (Poly-Ethylene Glycol, PEG-8000). Since different protein isoform(s)/transcript(s)/gene copy(s) are controlling the phenotype, it is crucial to identify genes specific to the favorable phenotype. The ensemble of genes, controlled by their own promotors with their own properties, steer the phenotype. The identified specific alleles can help to unravel the genetic basis attributing to drought tolerance in banana. Hence a workflow to study quantitatively the gene variants is indispensable. Allele- specific gene responses are picked up in this workflow as (1) non-identical isoforms matching the same gene locus, and (2) as products that are higher expressed in one cultivar than in others.

\section{Results}

Assessment of root biomass under control and osmotic stress. The growth of three different genotypes under control and osmotic stress (5\% PEG) conditions is observed after 21 days (Supplementary Fig. S1). Cachaco showed a relative growth $41.5 \%$ and $60.5 \%$ higher than the median of all control and stressed plants, respectively (Fig. 1). Cachaco has a significantly higher root growth than Grande Naine and Mbwazirume under $0 \%$ and $5 \%$ PEG conditions (Tukey HSD test, $\alpha=0.05$ ).

The root proteome. To unravel the molecular mechanisms behind the observed phenotypes, 234,739 features were aligned and quantified, 70,899 were targeted for MS2 (Supplementary Fig. S2). 51,765 spectrum sequence matches were found by integration of three search engines (Mascot, $x$ !Tandem and MS-GF+) searching cultivar specific databases. In total 5,261 peptide sequences were successfully identified in 2,749 unique proteins. The majority of successful, peptide sequence identifications was based on results from all three search engines (4,143), 423 identifications were unique for the Mascot search engine, and 243 and 46 for $\mathrm{x}$ !Tandem and MS-GF+, respectively (Supplementary Fig. S3). The identified proteins correspond to transcripts (mRNA) with relatively high abundance (Fig. 2). The peptides taken up in MS2, are generally found more in higher abundant spectra. MS1 peptides span 6 orders of magnitude 10, those targeted for MS2 3 orders, and the identified peptide sequences approximately 2 orders of magnitude (Supplementary Fig. S2). 
A

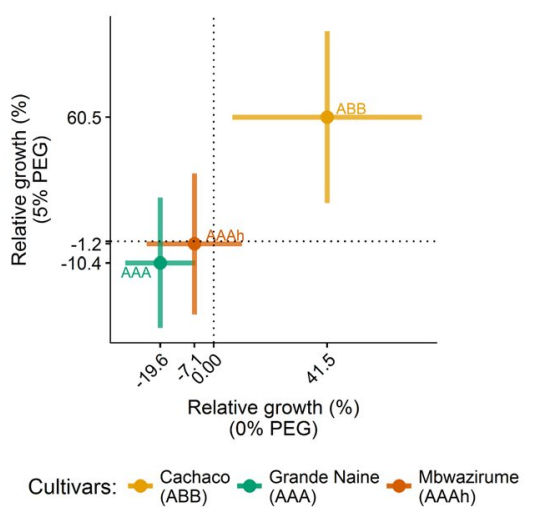

B

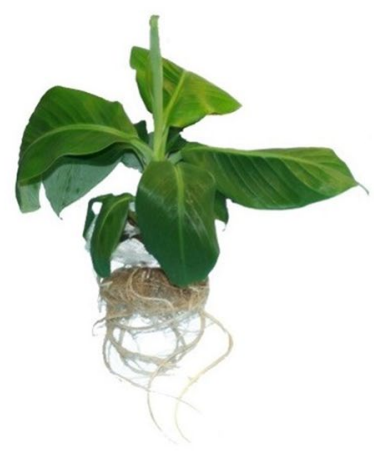

Figure 1. Relative root growth of AAA, AAAh, and ABB cultivars after 21 days of treatment ( 0 or $5 \%$ PEG. (A) Normalized dry weight of roots calculated by subtracting a plants dry weight from the treatment group median. Medians per cultivar are indicated with Inter Quartile Range. (B) Banana plant grown in the experimental setup.

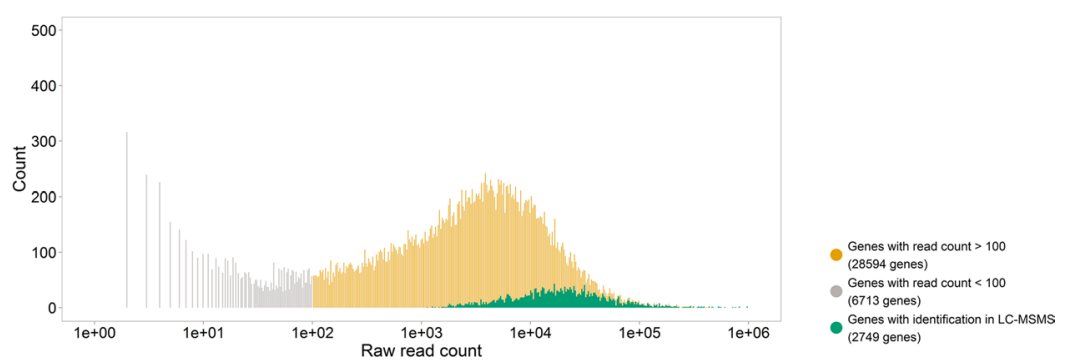

Figure 2. Identification of LC-MSMS spectra is correlated with the mRNA read count. Histogram with total read count in 18 samples visualized. Yellow: all genes in quantitative mRNA, green: all genes with identification in LC-MSMS, grey: genes with read counts below 100.
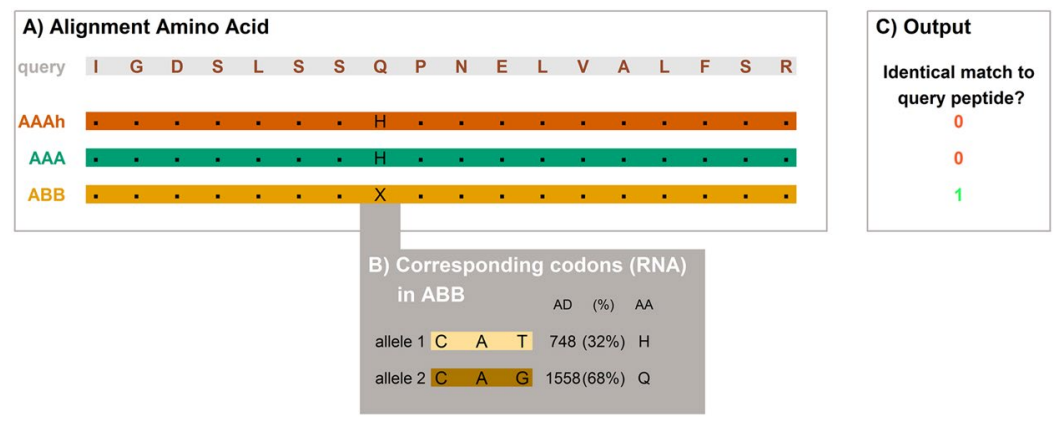

Figure 3. Integration of transcriptomics with proteomics to select cultivar specific alleles: assessment of aligned amino acid sequences and codons (RNA) reveals polymorphisms underlying the specific peptides. Exemplified for Ma02_t23730 (Sucrose Synthase 2). (A) Query peptide sequence identified in LC-MSMS (IGDSLSSQPNELVALFSR) aligned to AAA, AAAh, and ABB databases. (B) For the ambiguous amino acid in $\mathrm{ABB}$ database $(\mathrm{X})$ the variants of the corresponding codon are assessed in the RNA database of the cultivar of interest. The allele depth (AD) of 1558 (68\%) for allele 2 favors the presence of a unique amino acid (AA). (C) This is translated into specificity matching output.

Integration of proteomics with transcriptomics calls cultivar specific alleles. A locus (plural loci) in genetics is the position of a gene on a chromosome. The 5,261 validated feature sequences are spread over 2,749 loci (Supplementary Tables S1 and S2). A variant of the similar DNA sequence located at a given locus is called an allele. To call cultivar specific allelic sequences, all identified peptides were BLASTp searched across the 3 cultivar specific databases and the best match for each database was retained (Supplementary Table S2). The specificity of alignment is compared over the three cultivar specific databases. The workflow is exemplified for a query peptide (IGDSLSSQPNELVALFSR) identified in Ma02_t23730 (Sucrose Synthase 2; Fig. 3). A match on the same locus with less than $100 \%$ identity in another database identifies a cultivar specific peptide (Fig. 3A, Supplementary 


\begin{tabular}{|c|c|c|c|c|c|c|c|}
\hline Gene & Locus & Function & Variety & Sequence & Mascot & MS-GF+ & X!Tandem \\
\hline Ma02_t23730 & chr02:28633167-28639039 & sucrose synthase 2-like & Cach & IGDSLSSQPNELVALFSR & 92.11 & noMatch & noMatch \\
\hline Ma02_t23730 & chr02:28633167-28639039 & sucrose synthase 2-like & GN & IGDSLSSHPNELVALFSR & 20.63 & $1.66 \mathrm{E}-12$ & 0.00044 \\
\hline Ma02_t23730 & chr02:28633167-28639039 & sucrose synthase 2-like & Mbw & IGDSLSSHPNELVALFSR & 20.63 & $1.66 \mathrm{E}-12$ & 0.00044 \\
\hline Ma08_t33800 & chr08:44000904-44004899 & $\begin{array}{l}\text { phosphoglycerate kinase, } \\
\text { cytosolic-like }\end{array}$ & Cach & VDLNVPLDDNQKITDDTR & 40.79 & noMatch & noMatch \\
\hline Ma08_t33800 & chr08:44000904-44004899 & $\begin{array}{l}\text { phosphoglycerate kinase, } \\
\text { cytosolic-like }\end{array}$ & GN & VDLNVPLDDNLKITDDTR & 54.55 & $2.34 \mathrm{E}-11$ & 0.021 \\
\hline Ma08_t33800 & chr08:44000904-44004899 & $\begin{array}{l}\text { phosphoglycerate kinase, } \\
\text { cytosolic-like }\end{array}$ & Mbw & VDLNVPLDDNLKITDDTR & 54.55 & $2.34 \mathrm{E}-11$ & 0.021 \\
\hline
\end{tabular}

Table 1. Subset of the identified specific loci in 2 genes involved in glycolytic processes. The alternative cultivar specific sequences are provided. Identification scores from Mascot, msgf and x!Tandem search algorithms are given, noMatch: peptide not identified through this search algorithm.

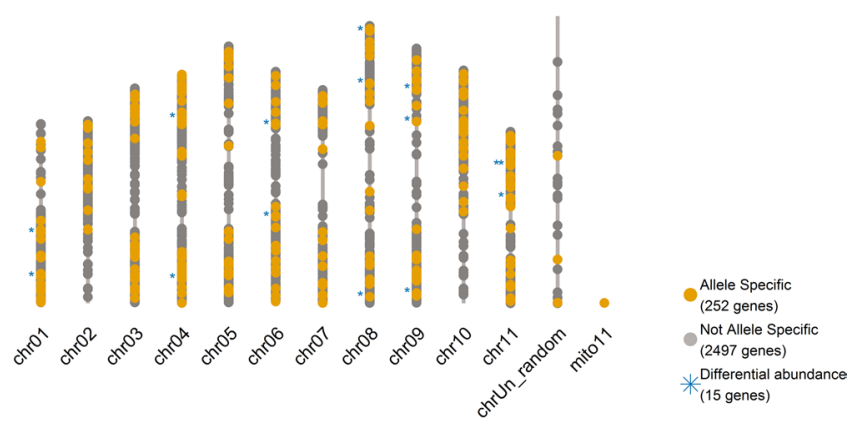

Figure 4. All identified gene loci, indicated when evidence (SAAP) for cultivar specificity was found. Differential peptide abundance between control (0\% PEG) and osmotic stress (5\% PEG) was assessed using the Kruskal Wallis test $(\alpha=0.1)$ in the ABB and AAA cultivars using 3 or 6 biological replicates respectively.

Fig. S4). The possibilities underlying the ambiguous amino acid X, were assessed at the transcriptome level (2 possible codons) at the putative SAAP location (Fig. 3B, Supplementary Fig. S4). The specific read count for the codons revealed whether or not the query amino acid was to be found at this locus. In this example case the query could be found, as such an ABB specific peptide sequence was identified (Fig. 3C).

Following this workflow (Supplementary Fig. S4), the integration of proteomics with transcriptomic validation identifies 252 loci with allelic isoforms. This is exemplified for 2 genes annotated as involved in glycolytic processes in Table 1, and can be found in Supplementary Table S3 for all the allele- specific proteins.

Figure 4 presents an overview of the loci identified with sequence specificity. 15 loci were linked to a differential abundance of the peptides in 0\% PEG relative to 5\% PEG samples (Kruskal-Wallis test, $\alpha=0.1$ ) (Fig. 4).

The relative abundance of allelic isoforms in the tolerant polyploid phenotype. The drought tolerant phenotype, Cachaco (ABB) carries two copies of the Musa balbisiana genome, and one of Musa acumi$n a t a^{29}$. Our workflow (Supplementary Fig. S4) identified both allele- specific protein sequences. The relative read distribution (mRNA) of these subgenome copies has been assessed and the read proportion is displayed as the ratio of $\mathrm{B}$ specific reads (Fig. 5A) over the total number of reads for the locus. The read proportion is distributed around 0.66 for the B alleles (Fig. 5B). 123 loci, dispersed all over the Musa genome, show intermediate read proportions not matching the expected $2 / 3^{\text {rd }}$ read distribution. In 32 loci, only one homeoallele was expressed (Supplementary Table S4).

Quantitative allele- specific expression in contrasting phenotypes. Based on the peptide intensity, a general quantitative overview of the root proteome displays the proteomic diversity between cultivars. Diversity between genomic subgroups controls the phenotypic divergence observed in Fig. 1. We are interested in mechanisms underlying the observed phenotypes. Sparse partial least squares discriminant analysis (sPLS-DA) discriminates genomic constitutions based on peptide abundance (ABB, AAAh, AAA) (Fig. 6A). Component 1 separates ABB from AAA samples, while component 2 separates Grande Naine and Mbwazirume, both AAA but originating from different subgroups of cultivated varieties: Cavendish and Mutika-Lugujira (East African Highland) bananas respectively. The result of sPLS-DA ranks different peptides in their specificity towards the cultivars. The combination of multivariate (sPLS-DA, horizontal axis, Fig. 6B) and univariate statistics (Kruskal Wallis, vertical axis; Fig. 6B) allows to identify peptides correlated with the drought tolerant phenotype (ABB specific, or AAA specific). Figure $6 \mathrm{C}$ and $\mathrm{D}$ show examples of the peptide abundance of AAA respectively ABB specific LC-MSMS features. This quantitative selection method identifies 131 additional genes with an ABB or AAA specific quantitative abundance profile, 29 of these are involved in glycolytic processes (Fig. 6, Table 2, see Supplementary Table S5 for all quantitatively selected specific proteins). 
A

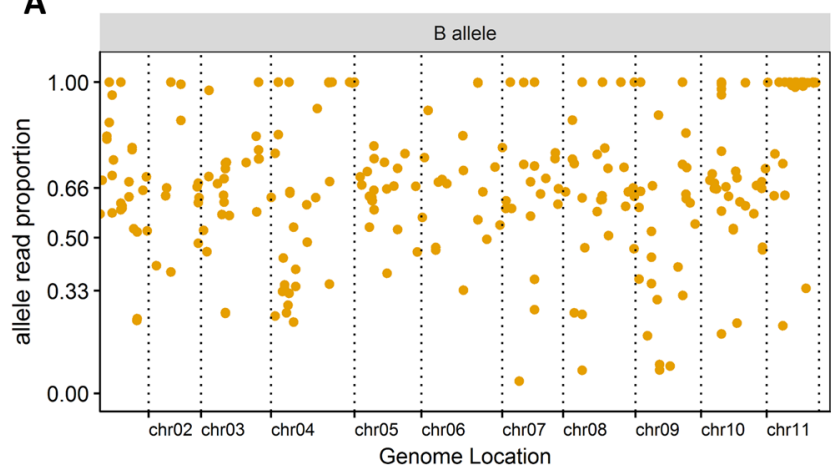

B

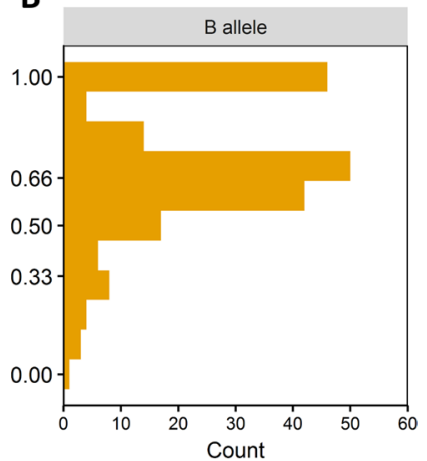

Figure 5. Allele- specific mRNA read proportions at SAAP locations (loci found in uniquely in ABB at the proteomic level) vary between 0 and 1 for the B alleles (A). Distribution pattern of the read proportion shows homeolog expression (bias) (B). mRNA reads assessed in ABB control samples, separated based on the RNA (codon) translation into $\mathrm{ABB}$ and non-ABB specific amino acid sequences.

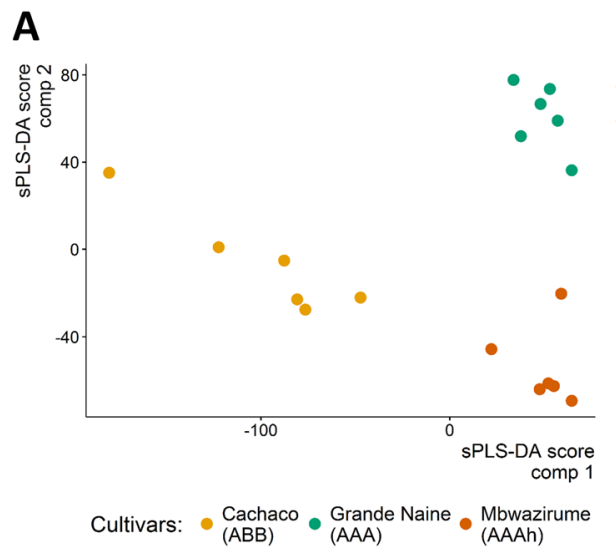

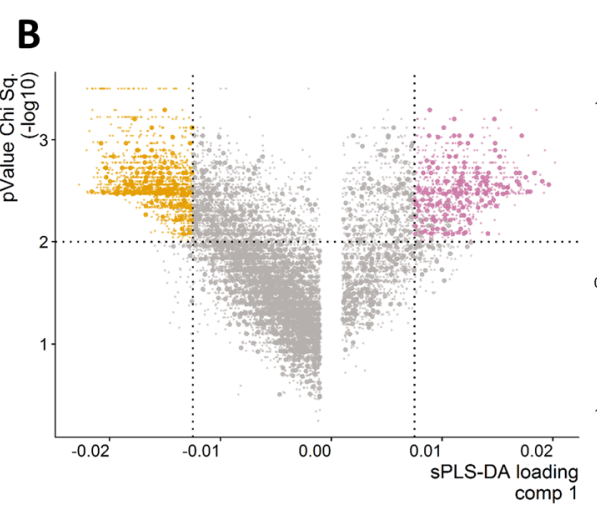

Specific abundance: $\bullet A A A \bullet A B B \bullet$ no

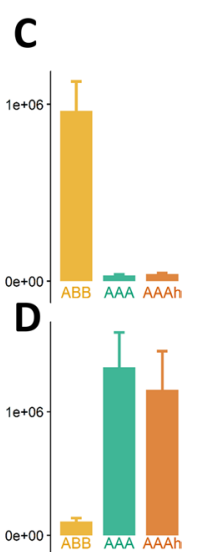

Figure 6. Quantitative selection of cultivar specific proteome features based on uni- and multivariate statistics. (A) Sparse partial least squares separates genomic groups (AAA and ABB) in Component 1. (B) Separation of features based on quantitative LC-MSMS. X-axis: sPLS-DA component 1: cut-off for selection $<-0.0125$ and $>0.0075$. Y-axis: $-\log 10$ of p-value of Chi Square Kruskal Wallis test with Benjamini-Hochberg p-value adjustment. P-value of 0.01 used as cutoff for Y axis. (C,D) Examples of ABB and AAA specific expression pattern respectively.

Functional annotation of alleles. 252 loci were found containing a SAAP, and an additional 131 loci with a phenotype specific abundance profile, without SAAP, were picked up. To characterize alleles correlated with enhanced root growth, we performed a gene ontology (GO) enrichment analysis on all allele- specific proteins. Gene ontology enrichment of these 383 loci relative to the 2,749 identified root proteins is shown in Supplementary Table S6. Energy related functions such as ATP metabolic process, nicotinamide nucleotide metabolic process, glycolysis and tricarboxylic acid cycle are enriched and correlate well with the observed growth phenotype.

\section{Discussion}

Characterization of polyploid non-model crops. Numerous studies have been undertaken to study the phenotypic and genetic variation of crops in relation to drought tolerance $e^{30-33}$. In this study we observe a distinct phenotype: Cachaco $(\mathrm{ABB})$ shows a significantly higher root dry mass compared to AAA cultivars, both under control and stress conditions ( $41.5 \& 60.5 \%$ relative to the median plant per treatment respectively; Fig. 1). Enhanced root growth is a drought avoidance/postponement mechanism and water uptake is (temporarily) ensured when deeper water layers are reached ${ }^{34}$. Typically deeper soil layers are less influenced by a decline in soil hydraulic conductivity in a drying environment ${ }^{35}$. Water uptake is determined by the exploration of a particular soil layer by the root biomass, and therefore deeper rooting varieties are possibly more drought tolerant by avoiding drought ${ }^{36}$. A minimal setback of root growth under variable soil moisture contents stabilizes plant performance during (limited) stress periods ${ }^{37,38}$. The main aim of this study was to pick up allele- specificity correlated to the favourable phenotype at the protein level in a relevant tissue and treatment. This eventually leads to better understanding of the genetic variation in a drought tolerant phenotype. 


\begin{tabular}{|c|c|c|c|c|c|c|c|}
\hline Gene & Locus & Function & Sequence & Specificity & $\begin{array}{l}\text { sPLS-DA } \\
\text { comp.1 (a) }\end{array}$ & pChi (b) & Groups (c) \\
\hline Ma07_t11500 & chr07:8539813-8543320 & Alcohol dehydrogenase-like 7 & IIGVDLNPDKFEIGK & AAAspec & 0.012196 & 0.00334 & b-a-a \\
\hline Ma05_t27700 & chr05:38886905-38891995 & $\begin{array}{l}\text { glyceraldehyde-3-phosphate } \\
\text { dehydrogenase 2, cytosolic }\end{array}$ & IVSNASCTTNCLAPLAK & ABBspec & -0.01322 & 0.003399 & $a-b-b$ \\
\hline Ma09_t02110 & chr09:1536831-1540788 & $\begin{array}{l}\text { glyceraldehyde-3-phosphate } \\
\text { dehydrogenase 2, cytosolic-like }\end{array}$ & IVSNASCTTNCLAPLAK & ABBspec & -0.01322 & 0.003399 & $a-b-b$ \\
\hline Ma08_t33830 & chr08:44031272-44038116 & $\begin{array}{l}\text { glyceraldehyde-3-phosphate } \\
\text { dehydrogenase GAPCP1, } \\
\text { chloroplastic-like }\end{array}$ & IVSNASCTTNCLAPLAK & ABBspec & -0.01322 & 0.003399 & $a-b-b$ \\
\hline Ma05_t00210 & chr05:152861-158287 & $\begin{array}{l}\text { glyceraldehyde-3-phosphate } \\
\text { dehydrogenase GAPCP2, } \\
\text { chloroplastic-like }\end{array}$ & IVSNASCTTNCLAPLAK & ABBspec & -0.01322 & 0.003399 & a-b-b \\
\hline Ma03_t32880 & chr03:34484915-34490528 & hexokinase-2-like & SDELFDFIASALVK & AAAspec & 0.012206 & 0.002754 & b-a-a \\
\hline Ma04_t16550 & chr04:15949921-15954721 & $\begin{array}{l}\text { malate dehydrogenase, } \\
\text { mitochondrial }\end{array}$ & ALEGSDVVIIPAGVPR & ABBspec & -0.02077 & 0.00108 & $a-c-b$ \\
\hline Ma04_t16550 & chr04:15949921-15954721 & $\begin{array}{l}\text { malate dehydrogenase, } \\
\text { mitochondrial }\end{array}$ & LNPLVSNLALYDIAGTPGVAADVGHINTR & ABBspec & -0.01539 & 0.002117 & $a-b-b$ \\
\hline Ma05_t03680 & chr05:2710087-2716309 & malate dehydrogenase-like & AGEWTIVQGLSVDEFSR & ABBspec & -0.014 & 0.006136 & $a-b-b$ \\
\hline Ma01_t19240 & chr01:14718937-14723088 & $\begin{array}{l}\text { phosphoglycerate kinase, } \\
\text { cytosolic, transcript variant X2 }\end{array}$ & ELDYLVGAVANPK & ABBspec & -0.0131 & 0.002117 & $a-b-b$ \\
\hline Ma05_t00310 & chr05:217490-221221 & $\begin{array}{l}\text { phosphoglycerate kinase, } \\
\text { cytosolic-like }\end{array}$ & ELDYLVGAVANPK & ABBspec & -0.0131 & 0.002117 & $a-b-b$ \\
\hline Ma05_t00310 & chr05:217490-221221 & $\begin{array}{l}\text { phosphoglycerate kinase, } \\
\text { cytosolic-like }\end{array}$ & LISALPDGGVLLLENVR & ABBspec & -0.01825 & 0.002754 & $a-b-b$ \\
\hline Ma11_t09970 & chr11:9163536-9171836 & $\begin{array}{l}\text { Putative Pyruvate kinase, } \\
\text { cytosolic isozyme }\end{array}$ & LGDLYQTQIFAK & ABBspec & -0.01351 & 0.003096 & $a-b-b$ \\
\hline Ma06_t14950 & chr06:10182092-10190553 & $\begin{array}{l}\text { Putative Pyruvate kinase, } \\
\text { cytosolic isozyme }\end{array}$ & LGDLYQTQIFAK & ABBspec & -0.01351 & 0.003096 & $a-b-b$ \\
\hline Ma00_t00620 & chrUn_random:1791125-1796085 & $\begin{array}{l}\text { Pyruvate kinase, cytosolic } \\
\text { isozyme }\end{array}$ & ANIDIDGILKELPNDGRVPK & ABBspec & -0.01532 & 0.002552 & $a-b-b$ \\
\hline Ma05_t31060 & chr05:41159306-41165776 & $\begin{array}{l}\text { triosephosphate isomerase, } \\
\text { cytosolic }\end{array}$ & VASADVVDVVVSPPFVFLPLVK & ABBspec & -0.01924 & 0.003321 & $a-b-b$ \\
\hline
\end{tabular}

Table 2. Selection of mass identical proteome features with cultivar specific abundance. Example data for proteins involved in glycolytic processes are shown. Feature selection is based on sparse partial least squares discriminant analysis (sPLS-DA) component 1 (a), Kruskal Wallis p-value (b), indicated by groups (Cachaco Grande Naine-Mbwazirume) (c), $\mathrm{a}>\mathrm{b}>\mathrm{c}$.

Integration of quantitative LC-MSMS proteomics with transcriptomics enables to call allelespecificity in a high-throughput way. Advanced whole genomic screening experiments enable the identification and interpretation of mutations at the genome level ${ }^{39}$. We developed a workflow to identify variants at the allele level, as our interest lies in the differential contributions of diverse subgenomes to the allopolyploid phenotype. Transcriptome as well as proteome- wide studies of these three contrasting phenotypes reveal differentiation between $\mathrm{ABB}$ and AAA transcripts and peptides in the first sPLS component whereas the differentiation between both AAA cultivars is taken up in the second component (Fig. 6) ${ }^{30}$. This characterizes the genetic diversity: within the three genotypes, both AAA resemble each other more closely than the ABB in these test conditions. These findings are convincing to study the differences between AAA and ABB in detail, at the allelic level. Polyploid crops are characterized by a multitude of gene variant $\times$ environment interactions. This heterogeneity makes quantitative, whole genome studies complex, but it is also the basis of the observed phenotypic differences. There is a need for workflows enabling the study of genetic heterogeneity, with respect to the unique alleles. At the same time allele- specificity calling provides an opportunity to look into the homeolog expression (bias).

Linkage studies between a preferential phenotype and nucleotide/amino acid polymorphisms identify genes putatively involved in the trait of interest. However, the sterile and polyploid nature of banana, where cultivars exist of many crossed inter specific hybrids, hamper the use of SNP mapping in association studies, especially with multi gene responses like abiotic stress tolerance ${ }^{12}$. Integration of proteomics with transcriptomics is a bottom-up approach, enabling allele- specificity calling, proteome- wide, in relevant tissues, time points and environmental conditions. The proteogenomics workflow elegantly proves the existence of 252 genome locations carrying polymorphisms between contrasting phenotypes. The combination of univariate and multivariate statistics on LC-MSMS abundances picks up allele- specific expression in another 131 genes, even without a causal SAAP at the genomic location (Fig. 6). Together they form (a subset of) the genetic basis for the phenotype of preference. Once validated in multiple cultivars the polymorphisms/locations are promising markers for selection for climate-smart cultivars, as they are evidenced at the protein level, and correlated to a more drought tolerant phenotype.

The impact of a tripled genome. After an allopolyploidy event two or more diverse genomes hybridize in a single organism. This implies the phenotype of this organism is characterized by a mosaic of different gene responses inherent to these diverse constituting genomes ${ }^{40,41}$. The genomes are regulated at the genetic, 
transcriptomic, proteomic, and epigenetic level, which results in a multitude of possibilities for expression levels and thus the phenotype ${ }^{42}$. The tolerant cultivar of interest in our study, Cachaco, is constituted by two Musa balbisiana and one Musa acuminata genomes ${ }^{29}$. In banana, it has been suggested that the presence of the Musa balbisiana genome contributes to drought tolerance, thus the specific B isoforms and the relative abundance thereof are of special interest ${ }^{25,32,43-46}$.

Several low throughput studies reported that the allelic variants in banana are not necessarily equally expressed ${ }^{25,26,41,46}$. Alleles act in part independently and every homeolog can contribute equally, or not, to the final phenotype. Homeolog expression bias favors allelic isoform(s) over another ${ }^{42}$. This suggests differential regulation and efficiency of the involved proteins at the allelic level, driving expression levels of genes and genomes to a preferential phenotype ${ }^{47}$. In the $\mathrm{ABB}$ cultivar, we expect allelic copies of a gene to be transcribed in relative proportions of $1 / 3$ and $2 / 3$ for the constituting $A$ and B alleles respectively. Nevertheless, positive and negative homeolog expression bias is observed at 123 loci (Fig. 5, Supplementary Table S4). Positive expression bias, loci where the relative read contribution for $B$ alleles is higher than 0.66 , identifies genes where the $B$ isoform is prevalently expressed in this specific time point and in this specific tissue. It is unclear what mechanism underlies the homeolog expression bias and how stable it is.

An extreme case of homeolog expression bias is called expression level dominance: the total gene expression is dominated by the expression of one of the homeologs, while the alternative variant is not expressed ${ }^{48,49}$. Previously, in a low throughput study the absence of the encoding gene sequence of an A homeolog of the Abscisic Acid stress ripening Asr-gene was already noted in our ABB banana ${ }^{46}$. This proves that expression level dominance occurs in triploid bananas, possibly due to backcrossing events during the formation of triploid banana cultivars $^{41}$. In our study, we found 32 genes that are characterized by expression level dominance: an allele- specific polymorphism unique to the ABB specific isoform (100\% relative read contribution) (Fig. 5, Supplementary Table S4). For example Ma11_t17540 (Glyceraldehyde 3 phosphate dehydrogenase), Ma11_t00520 (Aquaporin PIP2-3), Ma08_t09170 (Polyphenol oxidase). The AAA specific copy in these 32 genes is not actively transcribed. Expression level dominance could be the result of sub-/neofunctionalization of genes, but might also be tissue specific or time point specific expression, addressing the subgenomes in a different manner ${ }^{50}$. It might be the result of epigenetics, chromosome rearrangements, gene loss,... causing the non-favored gene (region) to be silenced or dropped.

Homeolog expression bias offers great flexibility to polyploid crops as this is not a static feature under various abiotic stresses. For example, a number of genes in the allopolyploid Coffea arabica cv. Java showed similar expression patterns compared with the parental C. canephora under hot conditions, while no parental preference was exhibited under cool conditions ${ }^{51}$. In a synthetic allotetraploid Arabidopsis, the ratio between expression levels of homeologs of the same loci changed significantly under cold stress in $1.11 \%$ of the identified homeologs ${ }^{52}$. GO enrichment revealed that the majority of these genes was involved in cold stress responses. These cases of homeolog expression bias under various environmental conditions exemplify the plasticity of allopolyploids and their relevance for agriculture ${ }^{53}$. However, not always can the link between homeolog expression bias and the environment be made at the genome level ${ }^{54}$. Genetic regulation in polyploids is complex. In similar subgenomes, the variations between homeologs are minor, and regulatory factors, like transcription factors, other binding factors, and epigenetic status, act independently of the subgenome ${ }^{52,54,55}$. Thus increased homeolog expression bias can highlight the local dissimilarity between subgenomes, and the specificity of one of the subgenomes to the prevailing environment.

ABB specific proteins are involved in drought tolerance related pathways. Functional genomic techniques (proteomics and transcriptomics) provide insights in a snapshot of the (metabolic) activities in root tissue under control and osmotic stress conditions at a specific time point. Under drought stress, the metabolic responses of roots and shoots are opposite: a relative investment in root metabolism coincides with a decreased growth metabolism in autotrophic shoots ${ }^{37,56}$. Roots are non-photosynthetic and depend entirely on respiration of assimilates synthesized aboveground. However, under (drought) stress conditions, an imbalance between photosynthesis and respiration occurs, hence energy efficiency at root level is interesting towards drought tolerance ${ }^{30,57,58}$.

In this study 383 gene loci were selected with sequence uniqueness or increased protein abundance specific for the cultivar displaying enhanced root growth. The functional annotation of these genes via gene ontology (GO) enrichment, shows that the identified allele- specific genes are a non-random subset of genes (Supplementary Table S6). There is a significant presence of proteins involved in pathways related to energy and metabolism, the glycolytic pathway for example (GO: 0006096, p-value 0.002). The Musa root response to osmotic stress is characterized by enhanced respiration, glycolysis and fermentation pathways ${ }^{30}$. Glycolysis is a major pathway which needs to produce reductants $(\mathrm{NAD}(\mathrm{P}) \mathrm{H})$ and carbohydrates for anabolic metabolism. Within the glycolytic pathway, 36 genes with a SAAP or quantitative cultivar specificity are identified (Table 1, Supplementary Table S3).

The identified specific genes are involved in general abiotic stress tolerance related pathways. Under higher respiratory status, attributed to osmotic stress, increased ROS production is inevitable. The mitochondrial electron transport chain is a major site of ROS production ${ }^{59,60}$. Specific polymorphisms are found within several genes involved in the maintenance of cellular integrity under (ROS)-stress (Supplementary Table S3, S5). NADP-isocitrate dehydrogenase for example produces NADPH which helps to keep small antioxidants in reduced state so that ROS can be scavenged ${ }^{59}$. Heat Shock Proteins (HSP) are another class of genes involved in chaperoning enzymes, and maintenance of proteins in their correct state. With gel- based proteomics in banana, Vanhove et al. ${ }^{26}$ identified specific isoforms of HSP70 at 6 different genomic locations, one of which upregulated under osmotic stress. Two HSP genes (Ma02_t18000, Heat shock cognate $70 \mathrm{kDa}$ protein, and Ma08_t21650, probable mediator of RNA polymerase II transcription subunit $37 \mathrm{c}$ ) are corresponding between our study and Vanhove et al. ${ }^{26}$. Using LC-MSMS we identify 6 additional SAAP specific isoforms within the Heat Shock Protein 
(HSP) family and 4 additional HSP genes with cultivar specific abundance pattern. We demonstrate that the integration of proteomics with transcriptomics in a relevant tissue (root) at a relevant time point (3 days) in a relevant condition (osmotic stress) allows high throughput identification of genes with specific isoforms.

In conclusion, this study succeeded to select allele- specific protein variants by integration of proteomics and transcriptomics, leading to further understanding of the genetic diversity in an allopolyploid non-model crop. The allopolyploid gene expression is governed by a plethora of gene products, inherent to the multitude of gene copies. Our workflow discriminates the drought tolerant ABB from drought susceptible AAA cultivars at the allelic level. This finally allows homeolog specific quantification of gene expression, promising to make most out of (polyploid) breeding and biodiversity characterization/evaluation.

\section{Methods}

Plant material, growth conditions and osmotic stress treatment. Banana plants of three genotypes representing important cultivated subgroups were selected: Cachaco (Bluggoe subgroup; ABB; ITC0643), Grande Naine (Cavendish subgroup; AAA; ITC0180), and Mbwazirume (Mutika-Lujugira subgroup; AAA; ITC0084). Plantlets were obtained through the International Musa Transit Center (ITC, Bioversity International, hosted at KU Leuven, Belgium) Plants were grown in $500 \mathrm{~mL}$ PP containers in a plant incubator (Aralab Fitoclima Bio 600) where lights were set in a cycle of $12 \mathrm{~h} / 12 \mathrm{~h}$ (light/dark), while the relative humidity and temperature were kept constant at $75 \%$ and $25^{\circ} \mathrm{C}$, respectively. Throughout plant growth the medium $(305 \mathrm{~mL})$ was renewed whenever the volume reached $55 \%$ of the original volume in at least one plant. The medium composition is: $361 \mathrm{mg} / \mathrm{L} \mathrm{KNO}_{3}$, $121 \mathrm{mg} / \mathrm{L} \mathrm{K}_{2} \mathrm{SO}_{4}, 176 \mathrm{mg} / \mathrm{L} \mathrm{MgSO}_{4} .7 \mathrm{H} 2 \mathrm{O}, 181 \mathrm{mg} / \mathrm{L} \mathrm{MgCl}_{2} \cdot 6 \mathrm{H}_{2} \mathrm{O}, 194 \mathrm{mg} / \mathrm{L} \mathrm{KH}_{2} \mathrm{PO}_{4}, 398 \mathrm{mg} / \mathrm{L} \mathrm{NaH}_{2} \mathrm{PO}_{4} \cdot 2 \mathrm{H}_{2} \mathrm{O}$, $464 \mathrm{mg} / \mathrm{L} \mathrm{Ca}\left(\mathrm{NO}_{3}\right)_{2} \cdot 4 \mathrm{H}_{2} \mathrm{O}, 105 \mathrm{mg} / \mathrm{L} \mathrm{CaCl} \cdot 2 \mathrm{H}_{2} \mathrm{O}, 60 \mathrm{mg} / \mathrm{L}$ Sequestrene, $1.1 \mathrm{mg} / \mathrm{L} \mathrm{H}_{3} \mathrm{BO}_{3}, 2.7 \mathrm{mg} / \mathrm{L} \mathrm{MnSO} \mathrm{M}_{4} \cdot \mathrm{H}_{2} \mathrm{O}$, $0.23 \mathrm{mg} / \mathrm{L} \mathrm{ZnSO}_{4} .7 \mathrm{H}_{2} \mathrm{O}, 0.16 \mathrm{mg} / \mathrm{L} \mathrm{CuSO}_{4} .5 \mathrm{H}_{2} \mathrm{O}, 0.07 \mathrm{mg} / \mathrm{L} \mathrm{NaMoO}_{4} .2 \mathrm{H}_{2} \mathrm{O}, \mathrm{pH}=6$ (modified from Swennen et al. ${ }^{61}$.

After 35 days of initial growth the stress treatment was initiated: the stressed subgroup received fresh medium including 5\% of PEG-8000, an osmotic stress agent mimicking drought stress ( $\mathrm{pF}$ 2.7). The control plants received a medium without addition of PEG-8000. 3 biological replicates per cultivar were harvested 3 days after the start of the 5\% PEG-8000 treatment. Root tips for proteomic analysis ( $\pm 4 \mathrm{~cm}$ long from the apex) were collected and the material was snap frozen in liquid nitrogen. 21 days after the start of the 5\% PEG treatment, 6 biological replicates of each cultivar were phenotyped, measuring fresh and dry weight (dried 14 days, at $80^{\circ} \mathrm{C}$ ) of the roots.

Protein extraction and data acquisition through mass spectrometry. Proteins were extracted following the phenol extraction/ammonium acetate precipitation protocol ${ }^{62,63}$. The digested samples $(1 \mu \mathrm{g} / 5 \mu \mathrm{L})$ were injected and separated on an Ultimate 3000 UPLC system (Dionex, Thermo Scientific, USA) equipped with a Acclaim PepMap100 pre column (C18 $3 \mu \mathrm{m}-100 \AA$, Thermo Scientific, USA) and an C18 PepMap RSLC $(2 \mu \mathrm{m}$, $50 \times 50 \mathrm{~cm}$, Thermo Scientific, USA) following a multiple step gradient with a linear gradient (flow rate: $0.300 \mu \mathrm{L} /$ $\mathrm{min}$ ): $4 \%$ buffer B ( $80 \%$ acetonitrile (CAN), in $0.08 \%$ formic acid (FA)) for $3 \mathrm{~min}, 4-12 \%$ in $17 \mathrm{~min}, 12-15 \%$ in $10 \mathrm{~min}, 15-18 \%$ in $45 \mathrm{~min}, 18-20 \%$ in $40 \mathrm{~min}, 20-30 \%$ in $65 \mathrm{~min}, 30-35 \%$ in $30 \mathrm{~min}, 35-65 \%$ in $10 \mathrm{~min}, 65-95 \%$ in $5 \mathrm{~min}, 95 \%$ for $40 \mathrm{~min}, 95-4 \%$ in $1 \mathrm{~min}, 4 \%$ for $39 \mathrm{~min}$. The Q Exactive Orbitrap mass spectrometer (Thermo Scientific, USA) was operated in positive ion mode with a nano spray voltage of $1.5 \mathrm{kV}$ and the source temperature set to $250^{\circ} \mathrm{C}$. ProteoMAss LTQ/FT-Hybrid ESI Pos. Mode Cal Mix (MSCAL5-1EA SUPELCO, Sigma Aldrich) was used as an external calibrant and the lock mass 445.12003 (polysiloxane) as an internal calibrant. The instrument was operated in data-dependent acquisition (DDA) mode with a survey MS scan at a resolution of 70,000 (fw hm at $\mathrm{m} / \mathrm{z} 200$ ) for the mass range of $\mathrm{m} / \mathrm{z} 400-1,600$ for precursor ions, followed by MS/MS scans of the top ten most intense peaks with $+2,+3$, and +4 charged ions above a threshold ion count of $1 \mathrm{e}^{+} 6$ at 17,500 resolution using normalized collision energy (NCE) of $25 \mathrm{eV}$ with an isolation window of $3.0 \mathrm{~m} / \mathrm{z}$, a dynamic exclusion of $30 \mathrm{~s}$, and an Apex Trigger of 5-15 s. All data were acquired with Xcalibur 2.2 software (Thermo Scientific, USA). For identification and alignment, all raw data were converted into mgf-files using Progenesis (v 4.1, Nonlinear Dynamics, UK).

Database construction and protein identification. Three cultivar specific databases were constructed based on mRNA seq reads from our plants (available at https://www.ncbi.nlm.nih.gov/bioproject/ PRJNA305241,30). This transcriptomic dataset was realigned and recounted to the updated reference genome ${ }^{14}$. RNA-seq reads, contained in FASTQ files, were first trimmed based on low-quality ends and adapters were removed with cutadapt, v 2.7.964. Quality was checked with FastQC (v0.11.3). Reads were then mapped against the Musa acuminata genome of reference (DH Pahang v2; http://banana-genome-hub.southgreen.fr/) ${ }^{14,65}$; using the splice junction mapper for RNA-seq STAR, v 2.5.0 ${ }^{66}$ with default parameters. Read groups were added for each alignment, reads were split using SplitNCigarReads and locally realigned with InderlRealigner, v 3.4 (Genome Analysis ToolKit ${ }^{67}$ ) resulting in sample specific Binary Alignment/Map (BAM) files. Finally cultivar specific databases were constructed by concatenating BAM files per cultivar (mpileup, SAMTools ${ }^{68,69}$ ), followed by variant calling, in reference to the Musa reference genome v2 (45,855 sequences) (bcftools, SAMTools) ${ }^{68,69}$. Each database was complemented by 116 common repository of adventitious proteins (CRAP, http://www.thegpm.org/ $\mathrm{crap} /$ ) and combined into a target/decoy database ${ }^{70}$ enabling False Discovery Rate (FDR) estimation. The decoy sequences were created by reversing the target sequences in SearchGUI v 3.1.4 $4^{71,72}$.

For every sample, peak lists (.mgf format) containing all MSMS spectra were extracted from Progenesis and were identified against its proper database integrating 3 database search algorithms: Mascot, v 2.2.06 ${ }^{73}$, X!Tandem, version X!Tandem Vengeance (2015.12.15.2) ${ }^{74}$, and MS-GF+, v 10282 ${ }^{75}$. The SearchGUI identification settings were as follows: enzyme trypsin, 2 missed cleavages (maximally), $10.0 \mathrm{ppm}$ as MS1 and $0.02 \mathrm{Da}$ as MS2 tolerance, Carbamidomethylation of $\mathrm{C}(+57.021464 \mathrm{Da})$ as fixed modification, Oxidation of $\mathrm{M}(+15.994915 \mathrm{Da})$, Acetylation of protein N-term $(+42.010565 \mathrm{Da})$, Pyrolidone from E (-18.010565 Da), Pyrolidone from Q 
$(-17.026549 \mathrm{Da})$, and Pyrolidone from carbamidomethylated C $(-17.026549 \mathrm{Da})$ as variable modifications. The search was not conducted with error tolerance.

Peptides and proteins were inferred from the spectrum identification results using PeptideShaker v 1.14.5 $5^{71}$. Peptide Spectrum Matches (PSMs), peptides, and proteins were validated at a 1.0\% False Discovery Rate (FDR) estimated using the decoy hit distribution. Results were exported and processed further in R software ( $\mathrm{v}$ 3.3.0) where PSMs were filtered, keeping the most confident peptide sequence matches. Only peptides identified in genes with average summed cultivar RNA seq read count above 100 were kept as trustworthy identifications. The mass spectrometry proteomics data have been deposited to the ProteomeXchange Consortium via the PRIDE partner repository with the dataset identifier PXD006375 and 10.6019/PXD006375 ${ }^{76}$.

Cross database allele- specific SAAP detection, validation, and quantification. To further validate the identified peptides, all peptides were searched across the 3 cultivar specific databases using the BLASTp algorithm $\mathrm{m}^{77}$ and the best match for each database was retained. This approach is performed at the locus level, implying that peptides matching multiple loci are evaluated separately. Allele- specific variants are identified as peptides matching exactly (100\%) in one database, while not in another database. In case of ambiguous proteins, containing amino acids depicted as X in the database, and caused by SNPs, inherent to the triploid banana nature, the underlying, possible mRNA variants were evaluated at this genome location. In these cases the uniqueness of sequence matching was evaluated by assessing the variant specific mRNA reads. ABB specific sequences, supposed to be the B alleles, are thus identified by their absence in AAA cultivars.

Quantifying homeoallele- specific reads was performed using Variant Call Format (VCF) files. These allowed to attribute the allele- specific read count to the identified allele- specific amino acid sequences in the control $(0 \%$ PEG) samples of the allopolyploid Cachaco (ABB). The VCF files are developed using the mRNA seq SNPs, and are called by UnifiedGenotyper (GATK), with the ploidy parameter set to 3. Only SNPs with a mapping quality superior to 30 were kept and SNP clusters (more than 2 SNPs in $10 \mathrm{bp}$ ) were discarded. This was bundled into a VCF-file containing all biological replicates. Based on proteomic sequence information the allele- specific read count was assigned to its coding variant sequence. For every validated ABB specific allele variant we tested if the observed read ratio corresponds to what is expected based on the genomic constitution. The ABB cultivar, Cachaco, carries 1 copy of the Musa acuminata subgenome, and 2 copies of the Musa balbisiana subgenome ${ }^{29}$, hence it is expected that the $A$ and $B$ variants are expressed in a $1 / 3^{\text {rd }}-2 / 3^{\text {rd }}$ ratio. This was tested by binomial testing using the number of $\mathrm{ABB}$ specific reads as number of successes, compared to the total number of reads for the specific locus, this with an expected ratio of 0.66 . P-value adjustment was according to Benjamini-Hochberg.

Multi- and univariate peptide statistics. Quantitative LC-MSMS was based on the peptide intensity using Progenesis. All samples were evaluated by Sparse Partial Least Squares Discriminant Analysis (sPLS-DA, mixOmics, R-package ${ }^{78}$ ), using genomic constitution groups (ABB, AAAh, AAA) as response variables. This multivariate statistical technique was complemented by the univariate, non-parametric Kruskal Wallis test $(\alpha<0.01$, p-value adjustment according to Benjamini-Hochberg; Agricolae, R-package).

Functional Gene Ontology enrichment. Musa gene annotations were taken over from the Universal Protein Resource (UniProt). Our gene identifiers were converted to UniProt standard format (http:// banana-genome-hub.southgreen.fr/convert; http://www.uniprot.org/uploadlists/) before Gene Ontology (GO) enrichment. We developed an in-house script to perform GO enrichment based on a user defined subset of genes in the UniProt format (https://labtrop.shinyapps.io/eRgo/). The tool is based on TopGO, R-package. Each molecular function (MF), biological process (BP) or cellular component (CC) is covered by a unique identifier, the GO ID. Data visualization as well as export of enriched GO terms are enabled in the tool. GO enrichment of all allele- specific proteins was performed against all 2,749 identified protein in the root proteome. We extracted all GO terms of biological process (BP) with significance level $\mathrm{p}<0.05$ (Fisher exact test).

\section{References}

1. Ortiz, R. \& Swennen, R. From crossbreeding to biotechnology-facilitated improvement of banana and plantain. Biotechnol. Adv. 32, 158-169 (2014)

2. van Asten, P. J. A., Fermont, A. M. \& Taulya, G. Drought is a major yield loss factor for rainfed East African highland banana. Agric. Water Manag. 98, 541-552 (2011).

3. Foley, J. A. et al. Solutions for a cultivated planet. Nature 478, 337-342 (2011).

4. Matthews, R. B., Rivington, M., Muhammed, S., Newton, A. C. \& Hallett, P. D. Adapting crops and cropping systems to future climates to ensure food security: The role of crop modelling. Glob. Food Sec. 2, 24-28 (2013).

5. Challinor, A. J. et al. A meta-analysis of crop yield under climate change and adaptation. Nat. Clim. Chang. 4, 287-291 (2014).

6. Calberto, G., Staver, C. \& Siles, P. In Climate change and food systems: global assessments and implications for food security and trade (ed. Elbehri, A.) (Food Agriculture Organization of the United Nations (FAO), 2015).

7. Langridge, P. \& Fleury, D. Making the most of 'omics' for crop breeding. Trends Biotechnol. 29, 33-40 (2011).

8. Scossa, F. et al. Genomics-based strategies for the use of natural variation in the improvement of crop metabolism. Plant Sci. 242, 47-64 (2016)

9. Perrier, X. et al. Multidisciplinary perspectives on banana (Musa spp.) domestication. Proc. Natl. Acad. Sci. USA 108, 11311-11318 (2011).

10. Sardos, J. et al. DArT whole genome profiling provides insights on the evolution and taxonomy of edible Banana (Musa spp.). Ann. Bot. 1-10 https://doi.org/10.1093/aob/mcw170 (2016).

11. De Buck, S. \& Swennen, R. Bananas the green gold of the South. VIB fact Ser. 1-54 (2016)

12. Sardos, J. et al. A Genome-Wide association study on the seedless phenotype in banana (Musa spp.) reveals the potential of a selected panel to detect candidate genes in a vegetatively propagated crop. PLoS One 11, 1-25 (2016).

13. Davey, M. W. et al. A draft Musa balbisiana genome sequence for molecular genetics in polyploid, inter- and intra-specific Musa hybrids. BMC Genomics 14, 1-20 (2013). 
14. Martin, G. et al. Improvement of the banana 'Musa acuminata' reference sequence using NGS data and semi-automated bioinformatics methods. BMC Genomics 17, 1-12 (2016).

15. D'Hont, A. et al. The banana (Musa acuminata) genome and the evolution of monocotyledonous plants. Nature 488, 213-218 (2012).

16. Wu, W. et al. Whole genome sequencing of a banana wild relative Musa itinerans provides insights into lineage-specific diversification of the Musa genus. Sci. Rep. 6, 1-11 (2016).

17. Barker, M. S., Arrigo, N., Baniaga, A. E., Li, Z. \& Levin, D. A. On the relative abundance of autopolyploids and allopolyploids. New Phytol. 210, 391-398 (2016).

18. Soltis, D. E., Misra, B. B., Shan, S., Chen, S. \& Soltis, P. S. Polyploidy and the proteome. Biochim. Biophys. Acta - Proteins Proteomics 1864, 896-907 (2016).

19. Wendel, J. F. The wondrous cycles of polyploidy in plants. Am. J. Bot. 102, 1753-1756 (2015)

20. Koh, J. et al. Comparative proteomics of the recently and recurrently formed natural allopolyploid Tragopogon mirus (Asteraceae) and its parents. New Phytol. 196, 292-305 (2012).

21. Chen, X. et al. Detection and genotyping of restriction fragment associated polymorphisms in polyploid crops with a pseudoreference sequence: a case study in allotetraploid Brassica napus. BMC Genomics 14, 1-12 (2013).

22. Zivy, M. et al. The quest for tolerant varieties: the importance of integrating 'omics' techniques to phenotyping. Front. Plant Sci. 6, 1-11 (2015).

23. Page, J. T., Gingle, A. R. \& Udall, J. A. PolyCat: a resource for genome categorization of sequencing reads from allopolyploid organisms. G3 3, 517-525 (2013).

24. Khan, A., Belfield, E. J., Harberd, N. P. \& Mithani, A. HANDS2: accurate assignment of homoeallelic base-identity in allopolyploids despite missing data. Sci. Rep. 6, 1-8 (2016).

25. Carpentier, S. C. et al. The use of $2 \mathrm{D}$-electrophoresis and de novo sequencing to characterize inter- and intra-cultivar protein polymorphisms in an allopolyploid crop. Phytochemistry 72, 1243-1250 (2011).

26. Vanhove, A. C., Vermaelen, W., Swennen, R. \& Carpentier, S. C. A look behind the screens: Characterization of the HSP70 family during osmotic stress in banana, a non-model crop. J. Proteomics 119, 10-20 (2015).

27. Carpentier, S. \& America, T. In Plant Proteomics: Methods and Protocols (ed. Jorrin-Novo, J.) 1072, 333-346 (Springer Science + Business, 2014).

28. Hu, G. et al. Proteomics profiling of fiber development and domestication in upland cotton (Gossypium hirsutum L.). Planta 240, 1237-1251 (2014).

29. Christelová, P. et al. Molecular and cytological characterization of the global Musa germplasm collection provides insights into the treasure of banana diversity. Biodivers. Conserv. 1-24 https://doi.org/10.1007/s10531-016-1273-9 (2016).

30. Zorrilla-Fontanesi, Y. et al. Differential root transcriptomics in a polyploid non-model crop: the importance of respiration during osmotic stress. Sci. Rep. 6, 1-14 (2016).

31. Yoo, C. Y. et al. The Arabidopsis GTL1 transcription factor regulates water use efficiency and drought tolerance by modulating stomatal density via transrepression of SDD1. Plant Cell 22, 4128-4141 (2010).

32. Vanhove, A.-C., Vermaelen, W., Panis, B., Swennen, R. \& Carpentier, S. C. Screening the banana biodiversity for drought tolerance: can an in vitro growth model and proteomics be used as a tool to discover tolerant varieties and understand homeostasis. Front. Plant Sci. 3, 1-10 (2012).

33. Rizhsky, L., Liang, H. \& Mittler, R. The combined effect of drought stress and heat shock on gene expression in tobacco. Plant Phisiology 130, 1143-1151 (2002).

34. Mickelbart, M. V., Hasegawa, P. M. \& Bailey-Serres, J. Genetic mechanisms of abiotic stress tolerance that translate to crop yield stability. Nat. Rev. Genet. 16, 237-251 (2015).

35. Kage, H., Kochler, M. \& Stützel, H. Root growth and dry matter partitioning of cauliflower under drought stress conditions: Measurement and simulation. Eur. J. Agron. 20, 379-394 (2004).

36. Pinheiro, H. A., DaMatta, F. M., Chaves, A. R. M., Loureiro, M. E. \& Ducatti, C. Drought tolerance is associated with rooting depth and stomatal control of water use in clones of Coffea canephora. Ann. Bot. 96, 101-108 (2005).

37. Gargallo-Garriga, A. et al. Opposite metabolic responses of shoots and roots to drought. Sci. Rep. 4, 1-7 (2014)

38. Passioura, J. B. Phenotyping for drought tolerance in grain crops. Funct. Integr. Genomics 39, 851-859 (2012).

39. Ramu, P. et al. Cassava haplotype map highlights fixation of deleterious mutations during clonal propagation. Nat. Genet. 1-7 https://doi.org/10.1038/ng.3845 (2017).

40. Adams, K. L. \& Wendel, J. F. Polyploidy and genome evolution in plants. Curr. Opin. Plant Biol. 8, 135-141 (2005).

41. De Langhe, E., Hribova, E., Carpentier, S., Doleel, J. \& Swennen, R. Did backcrossing contribute to the origin of hybrid edible bananas? Ann. Bot. 106, 849-857 (2010).

42. Yoo, M. J., Szadkowski, E. P. \& Wendel, J. F. Homoeolog expression bias and expression level dominance in allopolyploid cotton. Heredity (Edinb). 110, 171-180 (2013).

43. Ekanayake, I., Ortiz, R. \& Vuylsteke, D. R. Influence of leaf age, soil moisture, VPD and time of day on leaf conductance of various Musa genotypes in a humid forest-moist savanna transition site. Ann. Bot. 74, 173-178 (1994).

44. Kissel, E., van Asten, P., Swennen, R., Lorenzen, J. \& Carpentier, S. C. Transpiration efficiency versus growth: Exploring the banana biodiversity for drought tolerance. Sci. Hortic. (Amsterdam). 185, 175-182 (2015).

45. Thomas, D. S., Turner, D. W. \& Eamus, D. Independent effects of the environment on the leaf gas exchange of three banana (Musa sp.) cultivars of different genomic constitution. Sci. Hortic. (Amsterdam). 75, 41-57 (1998).

46. Henry, I. M. et al. Structure and regulation of the Asr gene family in banana. Planta 234, 785-798 (2011)

47. Dong, S. \& Adams, K. L. Differential contributions to the transcriptome of duplicated genes in response to abiotic stresses in natural and synthetic polyploids. New Phytol. 190, 1045-1057 (2011).

48. Buggs, R. J. A. et al. The legacy of diploid progenitors in allopolyploid gene expression patterns. Philos. Trans. R. Soc. B Biol. Sci. 369, $1-13$ (2014).

49. Grover, C. E. et al. Homoeolog expression bias and expression level dominance in allopolyploids. New Phytol. 196, 966-971 (2012).

50. Yoo, M. J., Liu, X., Pires, J. C., Soltis, P. S. \& Soltis, D. E. Nonadditive Gene Expression in Polyploids. Annu. Rev. Genet 48, 485-517 (2014).

51. Bardil, A., de Almeida, J. D., Combes, M. C., Lashermes, P. \& Bertrand, B. Genomic expression dominance in the natural allopolyploid Coffea arabica is massively affected by growth temperature. New Phytol. 192, 760-774 (2011).

52. Akama, S., Shimizu-Inatsugi, R., Shimizu, K. K. \& Sese, J. Genome-wide quantification of homeolog expression ratio revealed nonstochastic gene regulation in synthetic allopolyploid Arabidopsis. Nucleic Acids Res. 42, 1-15 (2014).

53. Sattler, M. C., Carvalho, C. R. \& Clarindo, W. R. The polyploidy and its key role in plant breeding. Planta 243, 281-296 (2016).

54. Combes, M. C., Dereeper, A., Severac, D., Bertrand, B. \& Lashermes, P. Contribution of subgenomes to the transcriptome and their intertwined regulation in the allopolyploid Coffea arabica grown at contrasted temperatures. New Phytol. 200, 251-260 (2013).

55. Combes, M. C., Cenci, A., Baraille, H., Bertrand, B. \& Lashermes, P. Homeologous gene expression in response to growing temperature in a recent allopolyploid (Coffea arabica L.). J. Hered. 103, 36-46 (2012).

56. Pierik, R. \& Testerink, C. The art of being flexible: how to escape from shade, salt, and drought. Plant Physiol. 166, 5-22 (2014).

57. Flexas, J., Bota, J., Galmés, J., Medrano, H. \& Ribas-carbo, M. Keeping a positive carbon balance under adverse conditions: responses of photosynthesis and repiration to water stress. Physiol. Plant. 127, 343-352 (2006). 
58. Shu, L. et al. Genetic, proteomic and metabolic analysis of the regulation of energy storage in rice seedlings in response to drought. Proteomics 11, 4122-4138 (2012).

59. Møller, I. M. Plant Mitochondria and Oxidative Stress: Electron Transport, NADPH Turnover, and Metabolism of Reactive Oxygen Species. Annu. Rev. Plant Physiol. Plant Mol. Biol. 52, 561-591 (2001).

60. Carpentier, S. C. et al. Sugar-mediated acclimation: The importance of sucrose metabolism in meristems. J. Proteome Res. 9 , 5038-5046 (2010).

61. Swennen, R., De Langhe, E., Janssen, J. \& Decoene, D. Study of the root development of some Musa cultivars in hydroponics. Fruits 41, 515-524 (1986).

62. Buts, K. et al. Improving the identification rate of data independent label-free quantitative proteomics experiments on non-model crops: A case study on apple fruit. J. Proteomics 105, 31-45 (2014).

63. Carpentier, S. C. et al. Preparation of protein extracts from recalcitrant plant tissues: An evaluation of different methods for twodimensional gel electrophoresis analysis. Proteomics 5, 2497-2507 (2005).

64. Martin, M. Cutadapt removes adapter sequences from high-throughput sequencing reads. EMBnet.journal 17, 10-12 (2011)

65. Droc, G. et al. The Banana Genome Hub. Database 2013, 1-14 (2013).

66. Dobin, A. et al. STAR: Ultrafast universal RNA-seq aligner. Bioinformatics 29, 15-21 (2013).

67. McKenna, A. et al. The genome analysis toolkit: a MapReduce framework for analyzing next-generation DNA sequencing data. Genome Res. 20, 1297-1303 (2010).

68. Li, H. A statistical framework for SNP calling, mutation discovery, association mapping and population genetical parameter estimation from sequencing data. Bioinformatics 27, 2987-2993 (2011).

69. Li, H. et al. The Sequence Alignment/Map format and SAMtools. Bioinformatics 25, 2078-2079 (2009)

70. Elias, J. E. \& Gygi, S. P. Target decoy search strategy for mass spectrometry based proteomics. Methods Mol. Biol. 604, 55-71 (2010).

71. Vaudel, M. et al. PeptideShaker enables reanalysis of MS-derived proteomics data sets. Nat. Biotechnol. 33, 22-24 (2015).

72. Vaudel, M., Barsnes, H., Berven, F. S., Sickmann, A. \& Martens, L. SearchGUI: An open-source graphical user interface for simultaneous OMSSA and X!Tandem searches. Proteomics 11, 996-999 (2011).

73. Perkins, D. N., Pappin, D. J. C., Creasy, D. M. \& Cottrell, J. S. Probability-based protein identification by searching sequence databases using mass spectrometry data. Electrophoresis 20, 3551-3567 (1999).

74. Craig, R. \& Beavis, R. C. TANDEM: Matching proteins with tandem mass spectra. Bioinformatics 20, 1466-1467 (2004).

75. Kim, S. \& Pevzner, P. A. MS-GF+ makes progress towards a universal database search tool for proteomics. Nat. Commun. 5, 1-10 (2014)

76. Vizcaíno, J. A. et al. 2016 update of the PRIDE database and its related tools. Nucleic Acids Res. 44, 447-456 (2016).

77. Altschul, S. F., Gish, W., Miller, W., Myers, E. W. \& Lipman, D. J. Basic local alignment search tool. J. Mol. Biol. 215, 403-410 (1990).

78. Cao, K. A. L., Boitard, S. \& Besse, P. Sparse PLS discriminant analysis: biologically relevant feature selection and graphical displays for multiclass problems. BMC Bioinformatics 12, 1-16 (2011).

\section{Acknowledgements}

The authors would like to thank Kusay Arat for the technical support at Sybioma, Edwige André and Saskia Windelinckx for the plant growth, Annick De Troyer, Els Thiry and Hien Do for isolating total RNA for RNA sequencing and protein extraction. This work was supported by the Bioversity International project 'Adding value to the ITC collection through molecular and phenotypic characterization', financed by the Belgian Development Cooperation, the CGIAR Research Program on Roots, Tubers and Bananas (RTB) and the USAID project AID-BFS-G-II-00002-11 'Reviving the plantain breeding program at IITA-International Institute for Tropical Agriculture'. Mathieu Rouard is gratefully acknowledge for the critical reading of the manuscript. COST Action Phenomenall FA 1306 is gratefully acknowledged for financing the presence at numerous interesting meetings about phenotyping and omics integration.

\section{Author Contributions}

R.S. and S.C. conceived and designed the experiments. J.v.W., Y.H., E.K. and N.C. performed the experiments. J.v.W. and S.C. analysed the data. J.v.W. and S.C. wrote the manuscript. R.S. and S.C. supervised the study. All authors reviewed and approved the final manuscript.

\section{Additional Information}

Supplementary information accompanies this paper at https://doi.org/10.1038/s41598-018-19684-5.

Competing Interests: The authors declare that they have no competing interests.

Publisher's note: Springer Nature remains neutral with regard to jurisdictional claims in published maps and institutional affiliations.

Open Access This article is licensed under a Creative Commons Attribution 4.0 International License, which permits use, sharing, adaptation, distribution and reproduction in any medium or format, as long as you give appropriate credit to the original author(s) and the source, provide a link to the Creative Commons license, and indicate if changes were made. The images or other third party material in this article are included in the article's Creative Commons license, unless indicated otherwise in a credit line to the material. If material is not included in the article's Creative Commons license and your intended use is not permitted by statutory regulation or exceeds the permitted use, you will need to obtain permission directly from the copyright holder. To view a copy of this license, visit http://creativecommons.org/licenses/by/4.0/.

(C) The Author(s) 2018 